\title{
Awareness of Colorectal Cancer and Attitude Toward Screening Among the Public in the Aseer region, Saudi Arabia
}

Mohammed Attieh Alzahrani (1)

Mohammed Abdullah Ahmed Alshehri (2)

Abdulbari Ahmed Bakheet Alzahrani (2)

Abdullah Nasser M. Shablahah (2)

Hajar Alhussain Ali Almozher (2)

Nada Ali Hussen Alqahtani (2)

Sara Ali Hussain Magrafi (2)

Abdulaziz Saad Alshahrani (3)

Awad S. Alsamghan (4)

(1) Assistant Professor, Department of Medicine, College of Medicine, King Khalid University, Abha, Kingdom of Saudi Arabia

(2) Medical Student, Department of Medicine, College of Medicine, King Khalid University, Abha, Kingdom of Saudi Arabia

(3) Department of Medicine, Najran University, Najran, Kindom of Saudi Arabia

(4) Department of Family \& Community Medicine, College of Medicine, King Khalid University, Abha, Kingdom of Saudi Arabia

Corresponding Author:

Dr. Mohammed Attieh Alzahrani

Assistant Professor, Department of Internal Medicine,

College of Medicine, King Khalid University

P.O Box 641, Abha, Kingdom of Saudi Arabia

Zip code: 61421

Tel: 00966504586134 MA

Email: mohammedalzahrani641@gmail.com;dr_mohdaz@hotmail.com

Received September 2020. Accepted October 2020. Published November 1, 2020.

Please cite this article as: Alzahrani et al. Awareness of Colorectal Cancer and Attitude Toward Screening Among the Public in the Aseer region, Saudi Arabia. Middle East J Intern Med 2020; 13(2): 20-28. DOI: 10.5742/MEJIM2020.93786

\begin{abstract}
Purpose: This study assessed population awareness of colorectal cancer and the attitude toward screening and its determinants in the Aseer region of Saudi Arabia.
\end{abstract}

Patients and methods: A descriptive cross-sectional approach was used to identify all accessible populations in the Aseer region of southern Saudi Arabia. Data were collected from participants using an online pre-structured questionnaire. The tool assessed participants' biodemographic data, medical and family history, awareness regarding colorectal cancer and attitude toward screening.

Results: A total of 1,296 respondents completed the survey. Participants' ages ranged from 18 to 65 years, with a mean age of $29.6 \pm 13.8$ years. The majority of participants were female $(66.7 \% ; 864)$. A total of $1,018(78.5 \%)$ of the participants had heard about colorectal cancer, and $648(50 \%)$ correctly defined it as large intestine cancer. Regarding symptoms, participants mostly reported abdominal pain $(80.3 \%)$, blood in the stool $(77.2 \%)$, and a change in bowel habits $(\mathbf{7 6 . 7 \%})$. A good awareness level was established in nearly one-fifth of the participants.
A total of 1,236 participants $(95.4 \%)$ reported that they would undergo screening if they had any risk factor for colorectal cancer. The main barrier to undergo screening was that participants did not have any symptoms $(31.7 \%)$.

Conclusion: This survey revealed that the public awareness of colorectal cancer in the Aseer region was overall poor. Participants had some knowledge regarding individual aspects but lacked knowledge of others. We recommend that healthcare staff should pay more attention to improving public awareness of the recommended screening for colorectal cancer.

Key words: Awareness; Cancer of the colon; Colonoscopy; Intestine large; Occult blood; Population health; Preventive health care; Risk factors 


\section{Introduction}

Colorectal cancer (CRC) is the third most common cancer worldwide and the second leading cause of cancer deaths in the United States $(1,2)$. There is considerable variation in CRC incidence internationally, with the rates registered in Asia, Africa, and South America being lower than those in North America, Oceania, and Europe, where the CRC risk factors obesity, diabetes, poor diet, physical inactivity, and smoking are more common $(3,4)$.

In Saudi Arabia, the cancer mortality statistics show a significant upward trend in recent years (5). According to the Saudi Cancer Registry (SCR), colorectal cancer was the most common cancer among the male population and the third most common cancer among the female population (6).

CRC has numerous risk factors, of which the most important are obesity, a sedentary lifestyle, smoking, excessive consumption of alcohol and red meat, a low-fiber and high-fat diet, a positive family history, and age $>50$ years(7-10). CRC can be asymptomatic for long or present with different symptoms, such as changes in bowel habits, chronic abdominal pain, gastrointestinal tract bleeding, fever, unexplained weight loss, malaise, and fatigue. Furthermore, some chronic conditions, such as inflammatory bowel disease, irritable bowel syndrome, infection, or hemorrhoids, may predispose patients to colorectal cancer (11-13).

Public awareness and knowledge are determinants of health behavior change, along with changes in the environment that give rise to them(14). Increasing the public awareness of CRC will help to improve peoples' health behaviors, including their willingness to undergo screening for cancer and related disorders, enabling early diagnosis and the lowering of the cancerrelated burden in the population. Heightened awareness will help to reduce preventable risk factors through lifestyle modification, which will reflect good community health.

The current survey aimed to assess public awareness of CRC and the attitude toward screening and its determinants in the Aseer region of southern Saudi Arabia.

\section{Material and Methods}

A descriptive cross-sectional approach was used to identify all accessible populations in the Aseer region of southern Saudi Arabia.

We constructed a survey tool after an intensive literature review and expert consultation. The tool was reviewed by a panel of three experts for content validity. Tool reliability was assessed using a pilot study of 25 participants with a reliability coefficient ( $\alpha$-Cronbach's) of 0.76 . The tool covered the following data: participants' biodemographic data, such as age, gender, place of residence, education, profession, and participants' family history of CRC and practice regarding screening for CRC. Awareness was assessed using ten questions covering the definition, signs, and symptoms of CRC, and the diagnostic methods and importance of screening for CRC. The tool was presented as an electronic pre-structured online questionnaire. We obtained approval from the Research Ethics Commit- tee, Deanship of Scientific Research, King Khalid University (HAPO-06-B-001) on 05 June 2020, approval no. ECM\#20200705. Consent was obtained from the study participants prior to study commencement. Persons aged 18 years or older in the Aseer region were invited to participate in the survey. All social media platforms were used to distribute the electronic questionnaire during the period from 1st April until 30th June 2020.

\section{Data analysis}

Each correct answer to an awareness question counted as one point, and the total sum of the discrete scores of the different items was calculated. We defined a score of less than $60 \%$ (14 points) of the maximum score, as poor awareness. Good awareness was defined as a score of more than $60 \%$ (15 points) of the maximum score. Descriptive analysis based on frequency and percent distribution was performed for all variables, including biodemographic data, awareness items, and participants' attitude toward screening. Cross tabulation was used to assess the level of awareness compared to participants' personal and medical data. Relations were tested using Pearson's chi-square test.

After data were extracted from the survey, they were reviewed, coded, and analyzed using SPSS Statistics for Windows, version 22 (IBM Corp., Armonk, NY, US). All statistical analyses were performed using two-tailed tests. A P-value $<0.05$ was considered to be statistically significant.

\section{Results}

The online survey was distributed to 1,500 individuals, of whom 1,296 responded and completed the questionnaire, corresponding to a response rate of $86.4 \%$. Participants' age ranged from 18 to 65 years with a mean age of 29.6 (standard deviation 13.8 years). The majority of participants were female $(66.7 \%)$. A total of $992(76.5 \%)$ were university graduates. A monthly income of less than 5000 SR was recorded among 854 (65.9\%) respondents, while $87 \%$ were free of any chronic health problems. A total of 418 (32.3\%) participants had genetic disorders, but only $6(0.5 \%)$ had a family history of CRC (Table 1$)$.

Table 2 shows participants' awareness of CRC. A total of $78.5 \%$ had heard about CRC, and 648 (50\%) correctly defined it as large intestine cancer. Many participants knew the frequently described symptoms such as abdominal pain (80.3\%), blood in the stool (77.2\%), and change in bowel habits (76.7 \%). Regarding the risk factors for CRC, $79.2 \%$ of participants were familiar with inflammatory bowel disorder, $62.2 \%$ had a positive family history, and about half of them associated with smoking and a high-fat diet. More than half (55.4\%) of participants agreed that CRC is treatable when diagnosed. As for screening methods, participants were most familiar with colonoscopy $(92 \%)$, followed by computed tomography colonography (77.2\%), and stool analysis (55.4\%). The vast majority $(94.1 \%)$ of participants indicated that screening is important for the early diagnosis of CRC. Overall, 20.4\% of participants had a good awareness of CRC.

Table 3 illustrates the participants' attitude toward CRC screening. A majority (95.4\%) reported that they would undergo screening if they had any risk factors for CRC. The main barriers to screening reported by the participants were that they did not have any symptoms (31.7\%) and had not been thinking 
about undergoing screening (18.7\%). About $44 \%$ of the participants had no specific reason not to participate in screening.

Regarding the sources of information on CRC, Figure 1 shows that these were mostly the internet and books (34\%), followed by social media (27.3\%) and study $(26.7 \%)$.

Table 4 shows the level of awareness of CRC compared to participants' biodemographic data and source of information. Good awareness was more common among those aged under 30 years $(24.3 \%)$ than in those above $50(10.7 \%)$, and this difference was statistically significant $(\mathrm{P}=.001)$. Also, $27.3 \%$ of males had good awareness compared to $16.9 \%$ of females $(\mathrm{P}$
$=.001)$. Good awareness was more frequent among those who lived in urban areas (23.4\%) compared to $15.2 \%$ of rural residents $(\mathrm{P}=.001)$. With regard to occupation, $45.5 \%$ of healthcare workers had good awareness levels compared to $11.1 \%$ of nonhealthcare workers $(\mathrm{P}=.001)$. Among those with a family history of genetic disorders, $25.8 \%$ had good awareness compared to $17.8 \%$ of others $(\mathrm{P}=.001)$, and good awareness was significantly more frequent among those ready to undergo screening for $\mathrm{CRC}$ than in those who were not $(\mathrm{P}=.041)$. Good awareness was highest (45.7\%) in those who had their information from study, compared to $32.1 \%$ of those who had it from doctors, and $4 \%$ of those who had no source of information $(\mathrm{P}=.001)$.

Table 1: Biodemographic data of participants $(n=1296)$ in survey on colorectal cancer awareness in the Aseer region, Saudi Arabia

\begin{tabular}{|c|c|c|c|}
\hline Biodemographic data & & $\begin{array}{c}\text { Participants } \\
\text { (n) }\end{array}$ & $\%$ \\
\hline \multirow{4}{*}{ Age (years) } & $<30$ & 898 & $69.3 \%$ \\
\hline & $30-39$ & 186 & $14.4 \%$ \\
\hline & $40-49$ & 156 & $12.0 \%$ \\
\hline & $>50$ & 56 & $4.3 \%$ \\
\hline \multirow{2}{*}{ Gender } & Male & 432 & $33.3 \%$ \\
\hline & Female & 864 & $66.7 \%$ \\
\hline \multirow{2}{*}{ Area of residence } & Urban & 822 & $63.4 \%$ \\
\hline & Rural & 474 & $36.6 \%$ \\
\hline \multirow{2}{*}{ Marital status } & Not married & 862 & $66.5 \%$ \\
\hline & M arried & 434 & $33.5 \%$ \\
\hline \multirow{4}{*}{ Educational level } & Primary school & 14 & $1.1 \%$ \\
\hline & Secondary school & 28 & $2.2 \%$ \\
\hline & High school & 262 & $20.2 \%$ \\
\hline & University/more & 992 & $76.5 \%$ \\
\hline \multirow{3}{*}{ Occupation } & Healthcare worker & 154 & $11.9 \%$ \\
\hline & Non-healthcare worker & 306 & $23.6 \%$ \\
\hline & Unemployed & 836 & $64.5 \%$ \\
\hline \multirow{3}{*}{ Monthly income } & $<5000 \mathrm{SR}$ & 854 & $65.9 \%$ \\
\hline & $5000-15000 S R$ & 340 & $26.2 \%$ \\
\hline & $>15000 \mathrm{SR}$ & 102 & $7.9 \%$ \\
\hline \multirow{9}{*}{ Do you have any chronic diseases? } & No & 1128 & $87.0 \%$ \\
\hline & Diabetes mellitus & 30 & $2.3 \%$ \\
\hline & Hypertension & 26 & $2.0 \%$ \\
\hline & Asthma & 58 & $4.5 \%$ \\
\hline & Hypothyroidism & 8 & $0.6 \%$ \\
\hline & Irritable bowel syndrome & 10 & $0.8 \%$ \\
\hline & Colon cancer & 13 & $1.0 \%$ \\
\hline & Crohn's disease/UC & 4 & $0.3 \%$ \\
\hline & Others & 19 & $1.5 \%$ \\
\hline \multirow{2}{*}{ Family history of any genetic disease } & Yes & 418 & $32.3 \%$ \\
\hline & No & 878 & $67.7 \%$ \\
\hline \multirow{2}{*}{ Family history of colon cancer } & Yes & 6 & $0.5 \%$ \\
\hline & No & 1290 & $99.5 \%$ \\
\hline
\end{tabular}

(Abbreviations: SR, Saudi Riyal; UC, Ulcerative colitis) 
Table 2: Awareness of colorectal cancer among survey participants $(n=1296)$ in the Aseer region, Saudi Arabia

Awareness

Participants (n)

$\%$

Heard about colorectal cance
Whatis colorectalcancer
Colorectalcanceris common

Symp toms of colorectalcancer

Risk factors of colorectalcancer

Colorectalcanceris treatablewhen diagnosed

Time to screen forcolorectalcancer

Early screening is important

Screeningforcolorectalcancerhelps in early diagnosis and treatment

Overallawareness
Yes

No

Stomach cancer

Small intestine cancer

Large intestine cancer

Don't know

Yes

No

Don't know

Abdominal pain

Change in bowel habits

Nause and vomiting

Yellowish skin or eyes

Blood in stool

Maybe asymptomatic

Don't know

Old age

Smoking

Inflammatory bowel

disease

Positive family history

High-fat diet

Bowel polyps

Don't know

Yes

No

Don't know

When symptoms appear

At age of 20 years

At age of 50 years

At age of 70 years

Don't know

Stool analysis

$\mathrm{X}$-ray

Colonoscopy

CT coloncgraphy

Ultrasound

Don't know

Yes

Ho

Don't know

Yes

No

Don't know

Poor

Good
1018

278

166

150

648

332

520

306

470

668

638

384

180

642

266

50

422

372

608

478

372

334

44

718

20

558

562

110

212

12

400

320

98

532

446

78

40

1184

12

100

1220

10

66

1032

264
$78.5 \%$

$21.5 \%$

$12.8 \%$

$11.6 \%$

$50.0 \%$

$25.6 \%$

$40.1 \%$

$23.6 \%$

$36.3 \%$

$80.3 \%$

$76.7 \%$

$46.2 \%$

$21.6 \%$

$77.2 \%$

$32.0 \%$

$6.0 \%$

$54.9 \%$

$48.4 \%$

$79.2 \%$

$62.2 \%$

$48.4 \%$

$43.5 \%$

$5.7 \%$

$55.4 \%$

$1.5 \%$

$43.1 \%$

$43.4 \%$

$8.5 \%$

$16.4 \%$

$0.9 \%$

$30.9 \%$

$55.4 \%$

$17.0 \%$

$92.0 \%$

$77.2 \%$

$13.5 \%$

$6.9 \%$

$91.4 \%$

$0.9 \%$

$7.7 \%$

$94.1 \%$

$0.8 \%$

$5.1 \%$

$79.6 \%$

$20.4 \%$ 
Table 3: Survey participants' $(n=1296)$ attitude toward colorectal screening, Aseer region, Saudi Arabia

Practice items

Participants

(n)

$\%$

Will you do the screening if you have any risk factor of colorectal cancer

Yes

1236

$95.4 \%$

No

60

$4.6 \%$

Which of the following reasons prevent you from undergoing early screening for colorectal cancer

None

Don't have symptoms

411

$31.7 \%$

Screening cost

Delay the screening

Colorectal cancer is not dangerous

Screening is not important

Didn't think about screeningbefore

Not advi sed by doctor to do

Fear of the diagnosis colorectal cancer

Feel emb arrassed and stressed

No doctor response 
Table 4: Survey participants' $(n=1296)$ level of awareness on colorectal cancer in relation to their biodemographic data and source of information

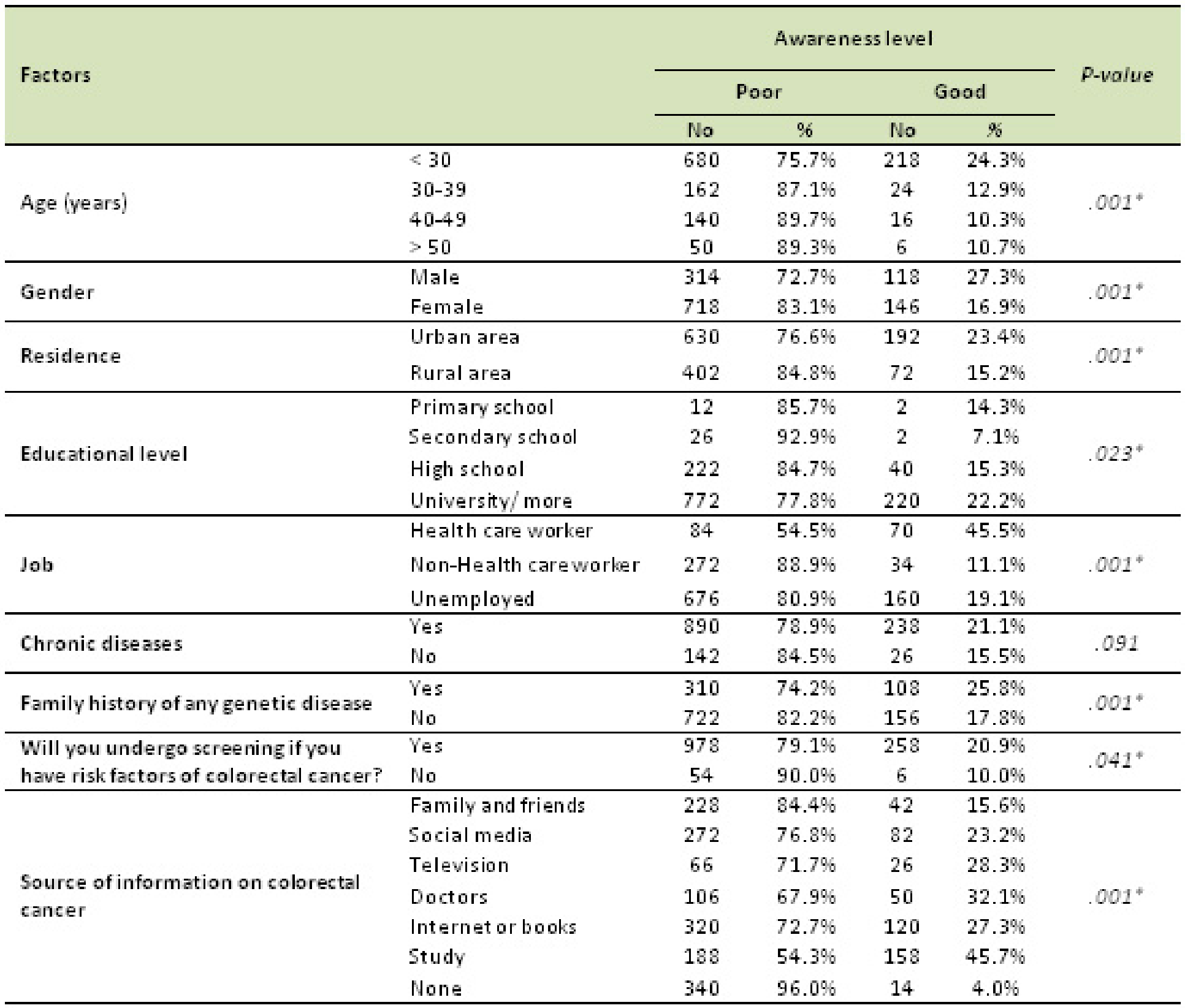

P: Pearson's chi-square test

$* \mathrm{P}<0.05$ (significant) 
Figure 1: Source of information on colorectal cancer among participants $(n=1296)$ in survey on awareness and knowledge on colorectal cancer, Aseer region, Saudi Arabia

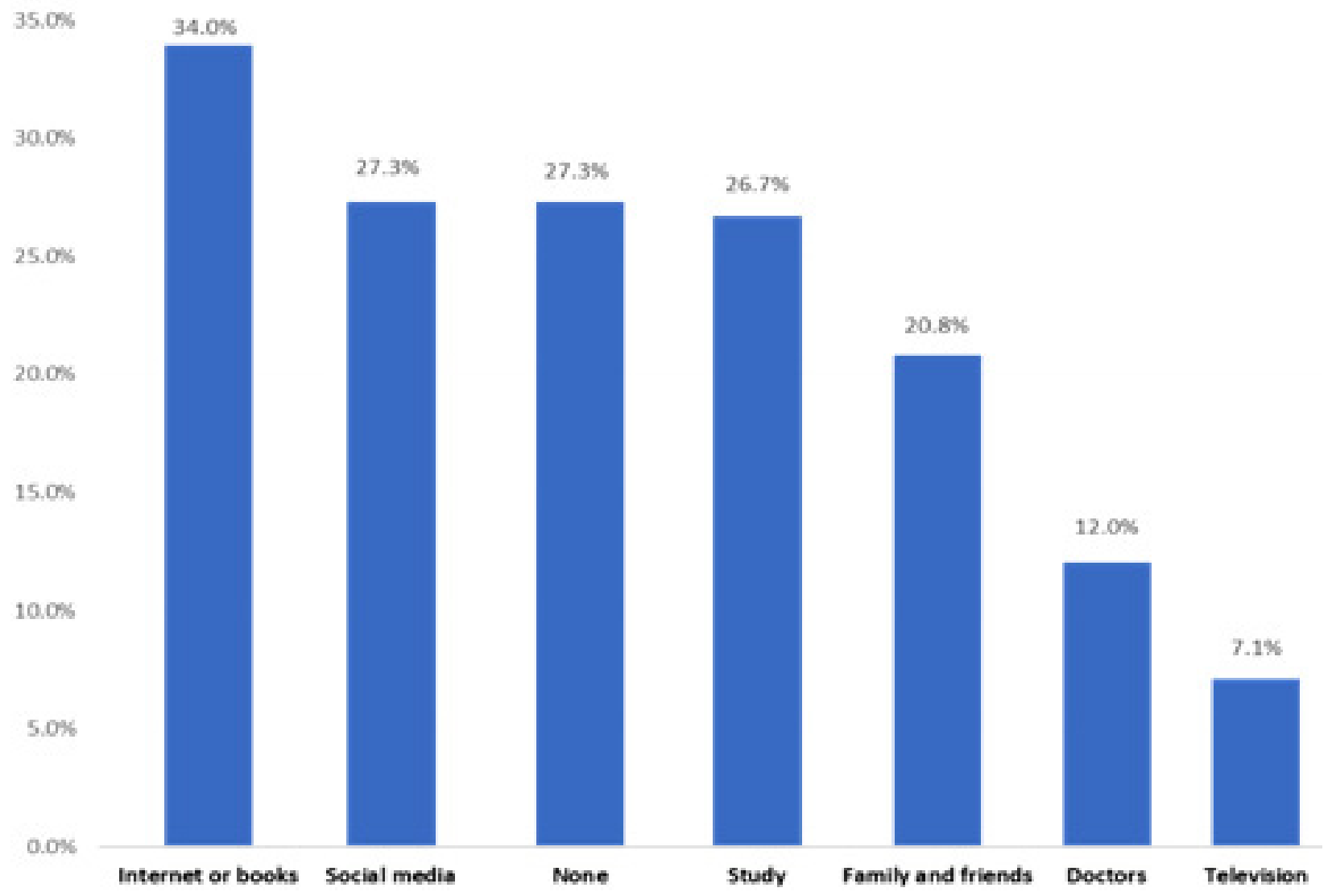

\section{Discussion}

This survey aimed to assess the awareness of CRC in the general population of the Aseer region in Saudi Arabia. Furthermore, the survey established participants' attitudes regarding CRC screening and the factors influencing this attitude. The results show that only one-fifth of the participants $(20.4 \%)$ were knowledgeable of CRC, but more than three-quarters had heard about CRC. Awareness was high for symptoms, particularly abdominal pain and change in bowel habits were well known, and the importance of screening and its role in successful treatment. Participants were also familiar with CRC risk factors, especially inflammatory bowel disease and a family history of CRC. About half of the participants knew that CRC is a treatable disease. Only $16 \%$ of participants knew the recommended age for starting screening, of 50 years.

One of the most interesting findings was that, while participants might be aware of individual items, their cumulative awareness was still poor. Participants were aware of some aspects of CRC but unaware of others. These results correspond to previous findings. In two other cross-sectional studies, screening awareness of CRC was more favorable $(47.9 \%$ and $83.0 \%$, respectively) $(15,16)$. Besides, $22 \%$ of the participants reported the frequency of CRC correctly, 59\% had acceptable information about the protocol, and $56.2 \%$ were informed about the development of CRC. In addition, $69.6 \%$ of the respondents reported the significance of early detection. Another report(17) showed a higher rate $(78.5 \%)$ of participants who were informed about the frequency and protocol of CRC screening, which was likely

linked to a relatively high level of education. Gede et al(18) conducted a survey assessing the awareness of CRC, its screening, and the sources of information in the Hungarian population. They reported that $32.7 \%$ of the participants were correctly informed about the recommended age for the beginning of CRC screening and $22.4 \%$ about its frequency. In our study participants, young age, male gender, high education levels, being a healthcare worker, and a family history of CRC were the most significant predictors for good awareness.

Moreover, physicians and studies as a source of information were significantly related to higher awareness than other sources. However, the internet and social media were used by more than half of the participants, whereas physicians were the primary source of information only in $12 \%$ of participants. This indicates that the physician's role as a health educator is not well established in Saudi Arabia. These findings were concordant with an Italian report, where participants' sources of information were primarily friends, followed by television and newspapers, and only thereafter general practitioners and specialists (19).

In Kuwait, Saeed et al (20) investigated the level of knowledge and awareness of CRC with regard to risk factors, symptoms, and screening/imaging procedures among the general public. Overall, $75 \%$ of the participants had heard about CRC, and the most reported risk factors were genetics and a family history of CRC (73.5\%). The most described symptoms were bloody stool, lower abdominal pain, obstructed intestine, and change in the nature of the exit, and the least common was anemia. Magnetic resonance imaging was identified as the most 
frequent diagnostic procedure (36\%), followed by computed tomography (31\%). On the other hand, $27.7 \%$ of participants had only limited knowledge about the different diagnostic procedures. In Saudi Arabia, a survey-based study(21) in Makkah revealed that most of the participants $(85.7 \%)$ had heard of CRC. However, nearly half of them had obtained their information as part of their school curriculum. More than half of the students knew that CRC might develop without any obvious symptoms. More than one-third (37.9\%) of participants believed that men were more likely than women to suffer from CRC, while almost one-third (29.4\%) said they knew nothing about the symptoms, and $21.8 \%$ thought that men and women have an equal chance of contracting CRC. The vast majority of participants $(92.2 \%)$ had never undergone screening for tumors of the colon and rectum. Only 3.6\% answered that they had undergone such screening. Another study(22) was conducted in Riyadh and revealed that most respondents believed that screening for CRC should begin with symptom onset $(42.9 \%)$. Less than $20 \%$ of respondents believed that polyps were a risk factor for $\mathrm{CRC}$, but the percentage varied significantly according to the level of education. However, even the most educated participants answered correctly less than $50 \%$ of the time. Similarly, only $34.8 \%$ of all respondents knew that a family history of CRC imparted a personal risk.

Among our participants, almost all (95\%) had intended to undergo screening if they had any risk factor. The main reasons for eventually not doing that were that they did not have any symptoms, they feared and delayed the screening, and, finally, the cost of screening, as nearly $65 \%$ of the respondents were not employed and had a monthly income of less than 5000 SR $(\$ 1,333)$.

\section{Conclusions}

In conclusion, our survey revealed that public awareness of CRC in the Aseer region was poor overall, but each participant had some knowledge of individual aspects while lacking knowledge of others. Our results also show that nearly all participants were ready to undergo screening for $\mathrm{CRC}$, but lacked knowledge regarding the recommended age for screening. The main barriers to screening were the absence of symptoms and risk factors, and the costs. We recommend that healthcare staff should pay more attention to improving public awareness of the recommended screening for chronic health problems, including CRC. This may happen in the form of short sessions of health education but should make use of posters, mass media, and social media, which were reported as the main source of information.

\section{Competing interest}

The author reports no conflicts of interest in this work.

\section{References}

1. International World Cancer Research Fund International. London: Colorectal cancer: WCRF International. 2012. Available from: http://www.wcrf.org/cancer_statistics/data_specific_cancers/colorectal_cancer_statistics.php . Accessed July 23, 2020.

2. Siegel RL, Fedewa SA, Anderson WF, et al. Colorectal cancer incidence patterns in the United States, 1974-2013. J Natl Cancer Inst. 2017;109(8):djw322.

3. Center MM, Jemal A, Smith RA, Ward E. Worldwide variations in colorectal cancer. CA Cancer J Clin. 2009;59(6):366378.

4. Haggar FA, Boushey RP. Colorectal cancer epidemiology: incidence, mortality, survival, and risk factors. Clin Colon Rectal Surg. 2009;22(4):191-197.

5. Ibrahim E, Sadiq BM, Banjar L, Awadalla S, Abomelha MS. Current and future cancer burden in Saudi Arabia: meeting the challenge. Hematol Oncol Stem Cel Ther. 2008;1(4):210-215.

6. Alyabsi M, Alhumaid A, Allah-Bakhsh H, Alkelya M, Aziz MA. Colorectal cancer in Saudi Arabia as the proof-of-principle model for implementing strategies of predictive, preventive, and personalized medicine in healthcare. EPMA Journal. 2020;11:119-131

7. Glade MJ. Food, nutrition, and the prevention of cancer: a global perspective. American Institute for Cancer Research/ World Cancer Research Fund, American Institute for Cancer Research, 1997. Nutrition. 1999;15(6):523-526.

8. Zisman AL, Nickolov A, Brand RE, Gorchow A, Roy HK. Associations between the age at diagnosis and location of colorectal cancer and the use of alcohol and tobacco: implications for screening. Arch Intern Med. 2006;166(6):629-634.

9. Boyle P, Langman MJ. ABC of colorectal cancer: Epidemiology. BMJ. 2000;321(7264):805-808.

10. Murphy N, Ward HA, Jenab M, et al. Heterogeneity of colorectal cancer risk factors by anatomical subsite in $10 \mathrm{Eu}-$ ropean countries: a multinational cohort study. Clin Gastroenterol Hepatol. 2019;17(7):1323-1331.

11. Adelstein BA, Macaskill P, Chan SF, Katelaris PH, Irwig L. Most bowel cancer symptoms do not indicate colorectal cancer and polyps: a systematic review. BMC Gastroenterol. 2011;11:65.

12. Astin M, Griffin T, Neal RD, Rose P, Hamilton W. The diagnostic value of symptoms for colorectal cancer in primary care: a systematic review. Br J Gen Pract. 2011;61(586):e231243.

13. Esteva M, Leiva A, Ramos M, et al. Factors related with symptom duration until diagnosis and treatment of symptomatic colorectal cancer. BMC Cancer. 2013;13:87.

14. World Cancer Research Fund International. London: New research on public awareness and knowledge of cancer prevention. Available from https://www.wcrf.org/int/blog/articles/2018/04/new-research-public-awareness-and-knowledgecancer-prevention. Accessed July 23, 2020. 
15. Tseng TS, Holt CL, Shipp M, et al. Predictors of colorectal cancer knowledge and screening among church-attending African Americans and Whites in the Deep South. J Community Health. 2009;34(2):90-97.

16. Papanikolaou IS, Sioulas AD, Kalimeris S, et al. Awareness and attitudes of Greek medical students on colorectal cancer screening. World J Gastrointest Endosc. 2012;4(11):513-517.

17. Sessa A, Abbate R, Di Giuseppe G, Marinelli P, Angelillo IF. Knowledge, attitudes, and preventive practices about colorectal cancer among adults in an area of Southern Italy. BMC Cancer. 2008;8:171.

18. Gede N, Kiss DR, Kiss I. Colorectal cancer and screening awareness and sources of information in the Hungarian population. BMC Fam Pract. 2018;19(1):106.

19. Domati F, Travlos E, Cirilli C, et al. Attitude of the Italian general population towards prevention and screening of the most common tumors, with special emphasis on colorectal malignancies. Intern Emerg Med. 2009;4(3):213-220.

20. Saeed RS, Bakir YY, Alkhalifah KH, Ali LM. Knowledge and awareness of colorectal cancer among general public of Kuwait. Asian Pac J Cancer Prev. 2018;19(9):2455-2460.

21. Al-Maghrabi AA. Assessment of the knowledge and awareness of colorectal cancer among general population of Makkah: a survey-based study. IJASR. 2016;4(4):168-175.

22. Zubaidi AM, AlSubaie NM, AlHumaid AA, Shaik SA, AlKhayal KA, AlObeed OA. Public awareness of colorectal cancer in Saudi Arabia: A survey of 1070 participants in Riyadh. Saudi J Gastroenterol. 2015; 21(2):78-83. 